\title{
Medium-spin states of the neutron-rich nucleus ${ }^{87} \mathrm{Br}$
}

\author{
B. M. Nyakó $\odot,{ }^{1}$ J. Timár $\odot,{ }^{1, *}$ M. Csatlós $\odot,{ }^{1}$ Zs. Dombrádi, ${ }^{1}$ A. Krasznahorkay $\odot,{ }^{1}$ I. Kuti, ${ }^{1}$ D. Sohler $\odot,{ }^{1}$ T. G. Tornyi $\odot,{ }^{1}$ \\ M. Czerwiński, ${ }^{2}$ T. Rząca-Urban $\odot,{ }^{2}$ W. Urban, ${ }^{2}$ P. Baczyk $\odot,{ }^{2}$ L. Atanasova $\odot,{ }^{3}$ D. L. Balabanski $\odot,{ }^{4}$ K. Sieja $\odot,{ }^{5,6}$ A. Blanc, ${ }^{7}$ \\ M. Jentschel, ${ }^{7}$ U. Köster, ${ }^{7}$ P. Mutti, ${ }^{7}$ T. Soldner, ${ }^{7}$ G. de France, ${ }^{8}$ G. S. Simpson, ${ }^{9}$ and C. A. Ur ${ }^{4}$ \\ ${ }^{1}$ Institute for Nuclear Research (Atomki), Pf. 51, 4001 Debrecen, Hungary \\ ${ }^{2}$ Faculty of Physics, University of Warsaw, ul. Pasteura 5, PL-02-093 Warsaw, Poland \\ ${ }^{3}$ Department of Medical Physics and Biophysics, Medical University-Sofia, 1431 Sofia, Bulgaria \\ ${ }^{4}$ ELI-NP, Horia Hulubei National Institute for $R \& D$ in Physics and Nuclear Engineering IFIN-HH, 077125 Bucharest-Magurele, Romania \\ ${ }^{5}$ Université de Strasbourg, IPHC, Strasbourg, France \\ ${ }^{6}$ CNRS, UMR7178, F-67037 Strasbourg, France \\ ${ }^{7}$ Institut Laue-Langevin, 71 Avenue des Martyrs, F-38042 Grenoble Cedex 9, France \\ ${ }^{8}$ GANIL, CEA/DSM-CNRS/IN2P3, Boulevard Henri Becquerel, BP 55027, F-14076 Caen Cedex 5, France \\ ${ }^{9}$ LPSC, Université Joseph Fourier Grenoble 1, CNRS/IN2P3, Institut National Polytechnique de Grenoble, F-38026 Grenoble Cedex, France
}

(Received 25 June 2020; accepted 22 February 2021; published 4 March 2021)

\begin{abstract}
Medium-spin excited states of the neutron-rich nucleus ${ }^{87} \mathrm{Br}$ were observed and studied for the first time. They were populated in fission of ${ }^{235} \mathrm{U}$ induced by the cold-neutron beam of the PF1B facility of the Institut Laue-Langevin, Grenoble. The measurement of $\gamma$ radiation following fission was performed using the EXILL array of Ge detectors. The observed level scheme was compared with results of large valence space shell model calculations. The medium-spin level scheme consists of three bandlike structures, which can be understood as bands built on the $\pi f_{5 / 2}, \pi\left(p_{3 / 2}+f_{5 / 2}\right)$, and $\pi g_{9 / 2}$ configurations. The behavior of the observed $\pi g_{9 / 2}$ band at high spins shows a considerable deviation from the shell model predictions. This deviation in this band is probably the result of an increased collectivity, which can be understood assuming that the $\pi g_{9 / 2}$ high- $j$ proton polarizes the core.
\end{abstract}

DOI: 10.1103/PhysRevC.103.034304

\section{INTRODUCTION}

Study of exotic, neutron-rich nuclei is in the forefront of contemporary nuclear-structure research. Such investigations have resulted in the observation of interesting new phenomena, like quenching of the known shell closures and formation of new ones. Thus, it is of special interest to study neutronrich nuclei near the shell closures. An interesting such region is that of the nuclei near the doubly magic exotic ${ }^{78} \mathrm{Ni}$. Besides the strength of the shell closures, the shell-model single-particle energies and residual interactions between the nucleons are also changing in these regions. Moreover, deformed intruder configurations could appear which are predicted to be pushed down in energy due to neutron-proton correlations with enhanced quadrupole collectivity. Indeed, the very recent results for ${ }^{78} \mathrm{Ni}$ indicate the breakdown of the neutron magic number 50 and proton magic number 28 beyond this stronghold, caused by a competing deformed structure [1]. Above $Z=28$ the first spectroscopy of lowlying levels in ${ }^{82,84} \mathrm{Zn}$ [2] at $N=52,54$ suggests that magicity is strictly confined to $N=50$ in ${ }^{80} \mathrm{Zn}$ with an onset of deformation developing towards heavier $\mathrm{Zn}$ isotopes. Excited states of odd-mass nuclei provide important information on the single-particle energies. However, very few medium-spin excited states are known for the odd-mass odd-proton nuclei

*Corresponding author: timar@atomki.hu with neutron number larger than 50 and proton number less than 37 near ${ }^{78} \mathrm{Ni}$. In general, information on excited mediumspin states in this region is very scarce, due to the difficulties in finding nuclear reactions in which these states could be populated with sufficient statistics.

In this work we aimed at studying the medium-spin states of the nucleus ${ }^{87} \mathrm{Br}$, which can be populated in the coldneutron induced fission of ${ }^{235} \mathrm{U}$ with relatively high yields. Using this reaction we can get closer to the doubly magic ${ }^{78} \mathrm{Ni}$. Preliminary results from our study on the excited states of the odd-proton ${ }^{87} \mathrm{Br}$ have been reported in a conference proceedings [3]. Several levels and tentative spin-parities of these results have been confirmed by a new study on the lowspin states of ${ }^{87} \mathrm{Br}$ from $\beta$ decay of ${ }^{87} \mathrm{Se}$, published recently [4]. The ground-state spin-parity has been assigned as $5 / 2^{-}$ in agreement with the tentative assignment of Ref. [5]. This assignment contradicts the earliest $3 / 2^{-}$assignment based on systematics, and raises the possibility of a deformed shape in this nucleus [6] providing a particular motivation to study also the deformation in ${ }^{87} \mathrm{Br}$. Recently, several studies have been reported on the low- and medium-spin states of some neighboring even-even, odd-odd, and odd-neutron nuclei [7-12].

\section{EXPERIMENTAL METHODS}

The experiment was performed using the PF1B coldneutron beam of the Institut Laue-Langevin (ILL) in Grenoble 
[13]. The neutron beam was shaped into a $12 \mathrm{~mm}$ diameter pencil beam with a thermal equivalence flux of $10^{8} /\left(\mathrm{s} \mathrm{cm}^{2}\right)$. In two parts of the beam time a $0.525 \mathrm{mg} / \mathrm{cm}^{2}$ and a $0.675 \mathrm{mg} / \mathrm{cm}^{2}$ thick ${ }^{235} \mathrm{U}$ target (both enriched to $99.7 \%$ ) were used, the former sandwiched between $15 \mu \mathrm{m}$ thick $\mathrm{Zr}$ backings and the second between $25 \mu \mathrm{m}$ thick Be backings, respectively, for rapid stopping of fission fragments. This enabled an almost Doppler-shift free measurement of the emitted $\gamma$ rays, which were detected by the EXILL detector array [14]. It consisted of eight Compton-suppressed EXOGAM Clover detectors [15], six Compton-suppressed GASP detectors [16] and two Clover detectors of the Lohengrin spectrometer [17]. The distance between the faces of detectors and target was about $15 \mathrm{~cm}$. The data were collected using a digital acquisition system with a $100 \mathrm{MHz}$ clock in a triggerless mode. Altogether 15 terabytes of data were collected over the 21 day period of the experiment. During the offline analysis the triggerless events, each consisting of an energy signal and the time of its registration, were arranged into coincidence events within various time windows (from 200 to $2400 \mathrm{~ns}$ ) and sorted into two- and three-dimensional histograms.

In order to calibrate the $\gamma$-ray energies in the coincidence matrices, we used inner calibration lines of known transitions strongly produced in the fission process. The used calibration lines were the 199.326(6), 330.88(9), 393.7(1), 431.3(1), and 509.3(1) $\mathrm{keV}$ transitions from ${ }^{144} \mathrm{Ba}$ [18]; the 588.825(18) $\mathrm{keV}$ transition from ${ }^{138} \mathrm{Xe}[19]$; the $815.0(1)$ and $977.8(1)$ $\mathrm{keV}$ transitions from ${ }^{96} \mathrm{Sr}$ [20]; the 1133.7(3) and 2247.8(2) $\mathrm{keV}$ transitions from ${ }^{135} \mathrm{I}$ [21]; as well as the 1279.0(1) and 2865.6(2) keV transitions from ${ }^{134} \mathrm{Te}$ [22]. The uncertainty of the calibration is $0.1 \mathrm{keV}$ below $1200 \mathrm{keV} \gamma$-ray energy and $0.3 \mathrm{keV}$ above it.

Information to assist in spin-parity assignment of the newly assigned levels could be inferred from the measured angular correlation relations of the subsequent transitions in the $\gamma$ decay cascades. The eight EXOGAM clover detectors were mounted in the EXILL spectrometer in one plane perpendicular to the beam direction in an octagonal geometry. The 28 detector pairs provided three different relative angles: $0^{\circ}$, $45^{\circ}$, and $90^{\circ}$ taking into account the symmetries. The way of analyzing of angular correlations in ${ }^{87} \mathrm{Br}$ is described in detail in Sec. 7.2 of Ref. [14]. In particular we took into account the fact that the EXILL clover detectors were operating in the add-back mode. For this reason the attenuation coefficients in the angular-correlation formula (for example see formula 3.3 in Ref. [23]), were carefully determined for the EXILL clover detectors, which cover larger solid angle than typical coaxial detectors. The relevant $\chi^{2}$-minimization procedure is illustrated in Fig. 17 of Ref. [14].

\section{LEVEL SCHEME}

In order to assign new transitions to ${ }^{87} \mathrm{Br}$ and to build the medium-spin level scheme of this nucleus, we analyzed the 3D $\gamma$-ray histograms created by applying a 200 ns coincidence time window and built in the Radware format [24]. The strategy we used for assigning new transitions to ${ }^{87} \mathrm{Br}$ was based on the fact that the transitions from the complementary fission fragments are in prompt coincidence with each other.

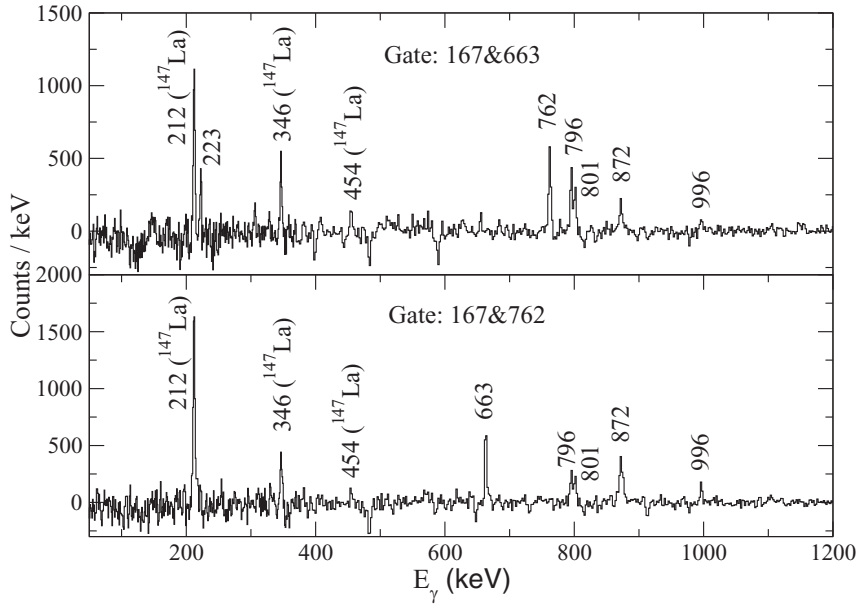

FIG. 1. Double-gated $\gamma \gamma \gamma$-coincidence spectra with one gate set on the strong $167 \mathrm{keV}{ }^{147} \mathrm{La}$ transition and the other gate set on one of the ${ }^{87} \mathrm{Br}$ candidate transitions.

In our case the main complementary fission fragments for ${ }^{87} \mathrm{Br}$ were the isotopes of Lanthanum. In the cold-neutron-induced fission of ${ }^{235} \mathrm{U}$ in most of the cases two or three neutrons and no protons were emitted from the primary fission fragments, leading, respectively, to ${ }^{147} \mathrm{La}$ and ${ }^{146} \mathrm{La}$, as secondary fission fragments. Fortunately, the level schemes of these nuclei are rather well known from previous fission experiments $[25,26]$. In the following we consider only these most probable types of the fission process.

As a first step, we have set double gates on several strong transition pairs of ${ }^{147} \mathrm{La}$ or of ${ }^{146} \mathrm{La}$. These spectra are expected to contain three types of $\gamma$ rays: (a) $\gamma$ rays belonging to ${ }^{147} \mathrm{La}$ or to ${ }^{146} \mathrm{La}$, respectively, (b) $\gamma$ rays belonging to the complementary fission fragments of ${ }^{147} \mathrm{La}$ or ${ }^{146} \mathrm{La}$, which are the ${ }^{86,87} \mathrm{Br}$ nuclei or the ${ }^{87,88} \mathrm{Br}$ nuclei, respectively, and (c) $\gamma$ rays belonging to other nuclei and being in coincidence with transitions having similar energies as the used gate pair.

The first type of $\gamma$ rays are known from previous studies $[25,26]$. The second type of $\gamma$ rays are expected to appear in all the gated spectra, while those of the third type are expected to appear only in one of them. Four large-intensity transitions have been seen to appear in most of the coincidence spectra with gate pairs from both the ${ }^{147} \mathrm{La}$ and the ${ }^{146} \mathrm{La}$ nuclei: the $619,663,762$, and $875 \mathrm{keV}$ transitions. Thus, these transitions are expected to belong to the ${ }^{87} \mathrm{Br}$ nucleus, which is the common complementary fission fragment of the two nuclei. This assignment is in agreement with the fact that none of these transitions are known to belong to the neighboring ${ }^{86,88} \mathrm{Br}$ nuclei, for which the medium-spin states are better known.

In the second step we set one of the double gates on one of these candidate transitions while the other gate on a strong transition of the complementary nucleus. Figure 1 shows examples of spectra obtained in such a way. In these spectra we observed other new transitions which are in coincidence with the candidate ${ }^{87} \mathrm{Br}$ transitions but do not belong to the complementary nucleus. Thus, these transitions probably belong also to ${ }^{87} \mathrm{Br}$. These transitions were very weak, or even not seen due to the background, in the first-step spectra. 


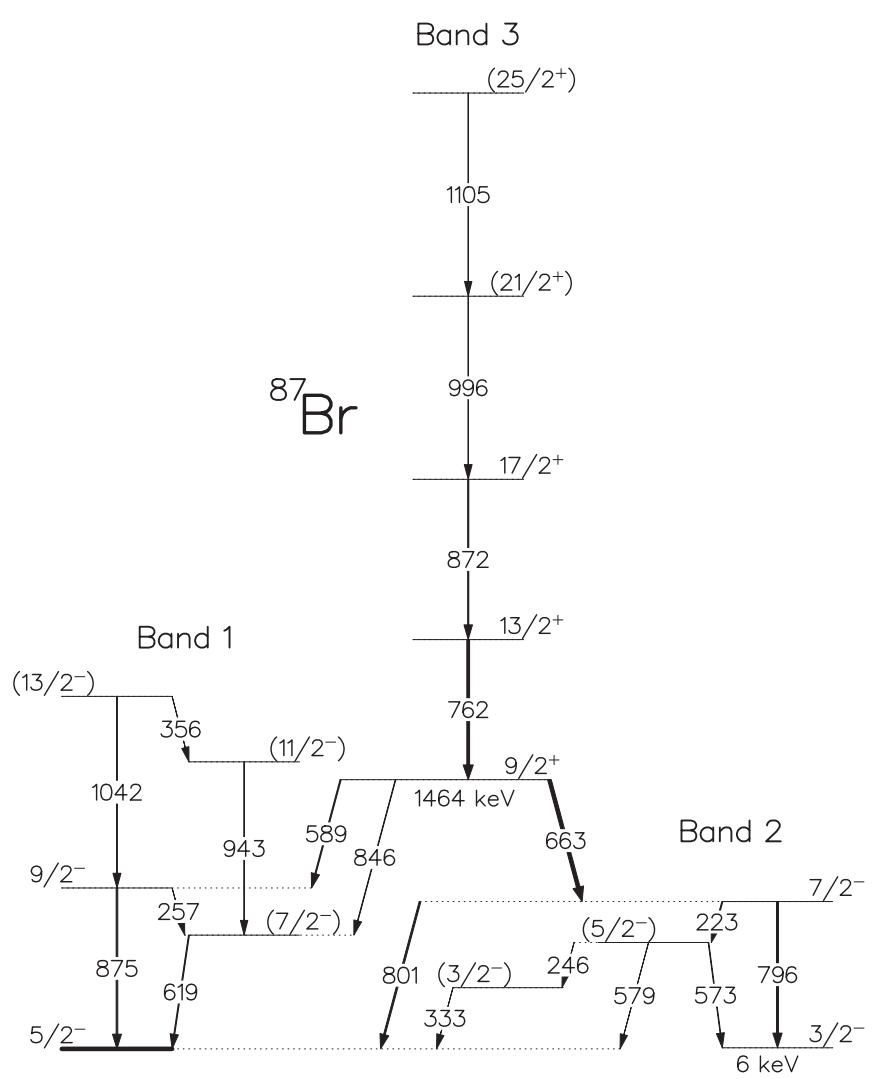

FIG. 2. Partial level scheme of ${ }^{87} \mathrm{Br}$, seen in prompt coincidence with ${ }^{146} \mathrm{La}$ and ${ }^{147} \mathrm{La}$. The energies are given in $\mathrm{keV}$, the widths of the transitions are proportional to their relative intensities.

Based on the coincidence relationships between these newly observed transitions, we could build a level scheme, plotted in Fig. 2.

This level structure does not correspond to any known level structure belonging to the populated fission fragments. Furthermore, when setting double gates on the strong transitions of this structure, we systematically see the strong transitions of the ${ }^{146} \mathrm{La}$ and ${ }^{147} \mathrm{La}$ nuclei in the spectra, as shown in Fig. 3 .

Based on these facts, we assign the obtained level scheme to ${ }^{87} \mathrm{Br}$. In this partial level scheme the levels correspond to the states populated from prompt fission. The major part of this partial level scheme has been reported in a conference proceedings [3]. In this level scheme we extended Bands 1 and 3 by one level each, and adopted the $333 \mathrm{keV}$ level from Ref. [4] compared to the level scheme in Ref. [3]. The levels up to the $1464 \mathrm{keV}$ one and the transitions depopulating them have been confirmed by Ref. [4]. The obtained level energies, spin-parities, $\gamma$-ray energies and relative intensities, as well as the $\gamma$-ray branching ratios are given in Table I.

In Table II we present results of angular-correlation analysis performed to help spin assignments of some of the excited levels in ${ }^{87} \mathrm{Br}$. Figure 4 illustrates such analysis for the $589-875 \mathrm{keV}$ cascade in ${ }^{87} \mathrm{Br}$. The upper panel shows the experimental $A_{2} / A_{0}$ and $A_{4} / A_{0}$ values with their uncertainties (blue box) compared against theoretical $\left(A_{2} / A_{0} ; A_{4} / A_{0}\right)$ points calculated for the mixing-ratios, $\delta$ ranging from minus infinity to plus infinity (Inf) represented by the ellipse (red). The green

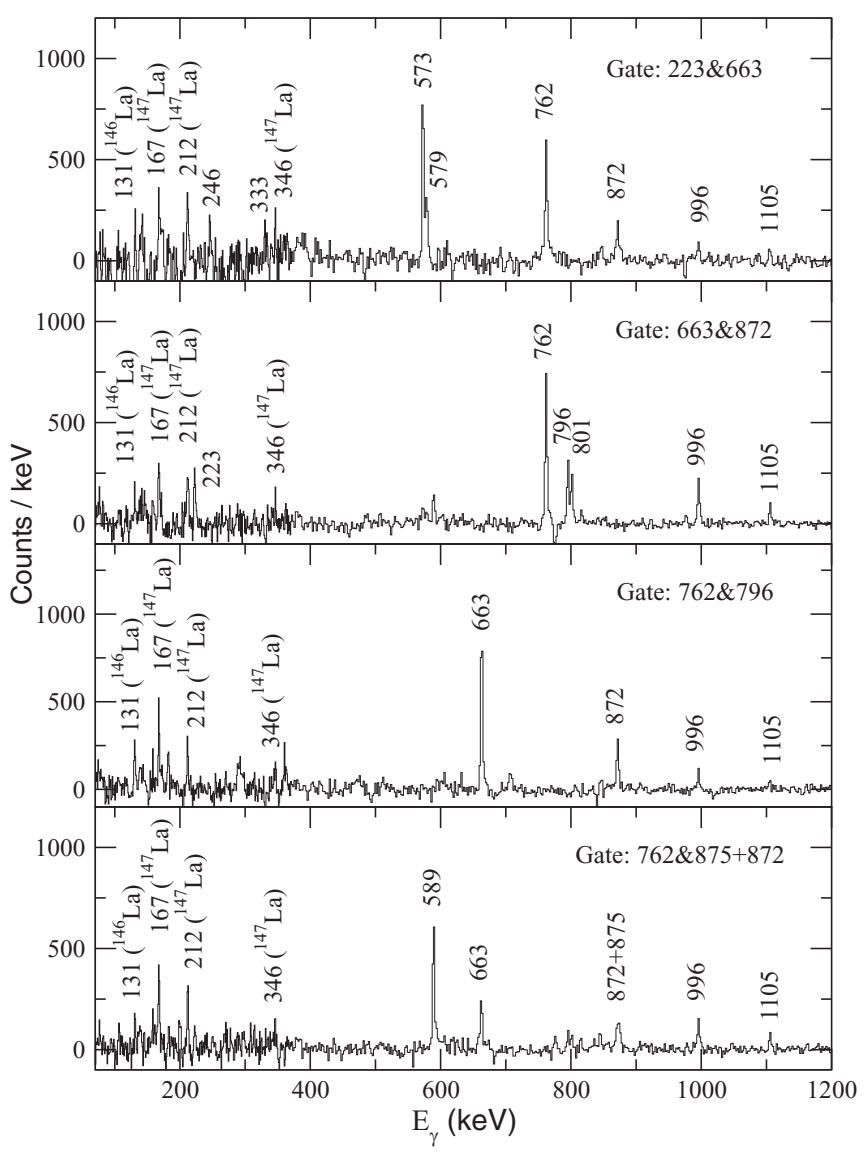

FIG. 3. Example double-gated $\gamma \gamma \gamma$-coincidence spectra confirming the placements of the newly observed ${ }^{87} \mathrm{Br}$ transitions in the level scheme.

cross shows the $\delta$ value of -0.456 determined by the $\chi^{2}$ analysis illustrated in the lower panel. The calculation was done for the $9 / 2 \rightarrow 9 / 2 \rightarrow 5 / 2$ spin hypothesis in the cascade. The theoretical $\left(A_{2} / A_{0} ; A_{4} / A_{0}\right)$ points for a stretched dipole or a stretched quadrupole $589 \mathrm{keV}$ transition are outside the experimental region. For example, in the pure stretched quadrupole case the expected $A_{2} / A_{0}$ and $A_{4} / A_{0}$ values are 0.102 and 0.009 , respectively.

The observed level scheme forms three bandlike structures labeled as Band 1, Band 2, and Band 3 in Fig. 2. We note that as Band 2 contains only three levels, these levels can also be nonband single-particle excitations. The bandheads of Band 1 and Band 2 are very close to each other in energy. The difference between them is only $6 \mathrm{keV}$. The bandhead of Band 1 is the ground state of ${ }^{87} \mathrm{Br}$. Two of the bands, Band 1 and Band 2, show the feature of strongly coupled $\Delta I=1$ rotational bands, while the third one, Band 3, looks like a weakly coupled or decoupled $\Delta I=2$ band.

The observed angular correlations of the two lowest transitions in Band 3, i.e., the 762 and $872 \mathrm{keV}$ transitions, are consistent with stretched quadrupole character for both of them. This indicates that Band 3 is an $E 2$ band, probably corresponding to a weakly coupled or decoupled configuration. This assumption is confirmed by the fact that no crossover transitions have been observed in this band. Both Band 1 and 
TABLE I. Level energies, spin-parities, $\gamma$-ray energies and relative intensities, as well as $\gamma$-ray branching ratios corresponding to the observed medium-spin level scheme of ${ }^{87} \mathrm{Br}$. The relative intensities are normalized to that of the strong $662.9 \mathrm{keV}$ transition. "nb" denotes a level not belonging to any band.

\begin{tabular}{lccrrrr}
\hline \hline$E_{i}$ & $I_{i}^{\pi}$ & Band & \multicolumn{1}{c}{$E_{f}$} & $E_{\gamma}$ & \multicolumn{1}{c}{$I_{\gamma}$} & $\mathrm{BR}$ \\
\hline 0.0 & $5 / 2^{-}$ & 1 & & & & \\
$6.0(3)$ & $3 / 2^{-}$ & 2 & & & & \\
$332.9(4)$ & $\left(3 / 2^{-}\right)$ & $\mathrm{nb}$ & 0.0 & $333.1(6)$ & $>2$ & \\
$578.8(2)$ & $\left(5 / 2^{-}\right)$ & 2 & 0.0 & $578.7(3)$ & $>7$ & $42(9)$ \\
& & & 6.0 & $572.8(3)$ & $>17$ & $100(9)$ \\
& & & 332.9 & $246.0(5)$ & $>2$ & $12(4)$ \\
$618.7(3)$ & $\left(7 / 2^{-}\right)$ & 1 & 0.0 & $618.9(4)$ & $>26$ & \\
$801.5(2)$ & $7 / 2^{-}$ & 2 & 0.0 & $801.4(3)$ & $46(5)$ & \\
& & & 6.0 & $795.5(3)$ & $54(5)$ & \\
& & & 578.8 & $222.7(3)$ & $26(4)$ & \\
$875.3(3)$ & $9 / 2^{-}$ & 1 & 0.0 & $875.2(5)$ & $>45$ & $100(17)$ \\
& & & 618.7 & $257.0(7)$ & $>5$ & $12(3)$ \\
$1464.4(3)$ & $9 / 2^{+}$ & 3 & 618.7 & $845.7(6)$ & $15(3)$ & \\
& & & 801.5 & $662.9(2)$ & $100(8)$ & \\
& & & 875.3 & $589.1(3)$ & $31(3)$ & \\
$1561.4(5)$ & $\left(11 / 2^{-}\right)$ & 1 & 618.7 & $942.7(5)$ & $>4$ & \\
$1917.4(6)$ & $\left(13 / 2^{-}\right)$ & 1 & 875.3 & $1042.0(6)$ & $19(3)$ & \\
& & & 1561.4 & $356.0(7)$ & $4(2)$ & \\
$2226.3(3)$ & $13 / 2^{+}$ & 3 & 1464.4 & $761.9(2)$ & $77(6)$ & \\
$3098.0(4)$ & $17 / 2^{+}$ & 3 & 2226.3 & $871.7(2)$ & $31(4)$ & \\
$4094.0(5)$ & $\left(21 / 2^{+}\right)$ & 3 & 3098.0 & $996.0(3)$ & $12(2)$ & \\
$5199.2(6)$ & $\left(25 / 2^{+}\right)$ & 3 & 4094.0 & $1105.2(3)$ & $7(2)$ & \\
\hline \hline
\end{tabular}

Band 2 look like $\Delta I=1$ bands with quadrupole crossover transitions. This assumption is also confirmed by the angular correlations observed for the 663-796 keV cascade in Band 2 and for the 589-875 keV cascade in Band 1. The observed angular correlations in the first cascade are consistent with the stretched quadrupole character of the $796 \mathrm{keV}$ transition and stretched dipole character of the $663 \mathrm{keV}$ transition. In Band 1, the observed angular correlations are consistent with the stretched quadrupole character of the $875 \mathrm{keV}$ transition and $\Delta I=0$ dipole character of the $589 \mathrm{keV}$ transition.

Using the multipole character of the transitions in ${ }^{87} \mathrm{Br}$ derived in this work and the unique spin-parity assignments reported in Ref. [4], together with information on the level schemes of neighboring odd-proton nuclei, we propose spinparity values for levels in ${ }^{87} \mathrm{Br}$ as shown in Table I and in Fig. 2. Our analysis agrees with Ref. [4] and provides unique spin-parity assignments for the 6,801, 2226, and $3098 \mathrm{keV}$ levels.

TABLE II. Normalized experimental angular correlation coefficients for selected $E_{\gamma 1}-E_{\gamma 2} \gamma$ cascades in ${ }^{87} \mathrm{Br}$.

\begin{tabular}{lrcr}
\hline \hline$E_{\gamma 1}-E_{\gamma 2}$ & \multicolumn{1}{c}{$A_{2} / A_{0}$} & \multicolumn{1}{c}{$A_{4} / A_{0}$} & Spin sequence \\
\hline $662.9-795.5$ & $-0.05(4)$ & $0.05(10)$ & $9 / 2 \rightarrow 7 / 2 \rightarrow 3 / 2$ \\
$589.1-875.2$ & $0.24(2)$ & $0.08(4)$ & $9 / 2 \rightarrow 9 / 2 \rightarrow 5 / 2$ \\
$871.7-761.9$ & $0.11(6)$ & $-0.09(13)$ & $17 / 2 \rightarrow 13 / 2 \rightarrow 9 / 2$ \\
\hline \hline
\end{tabular}
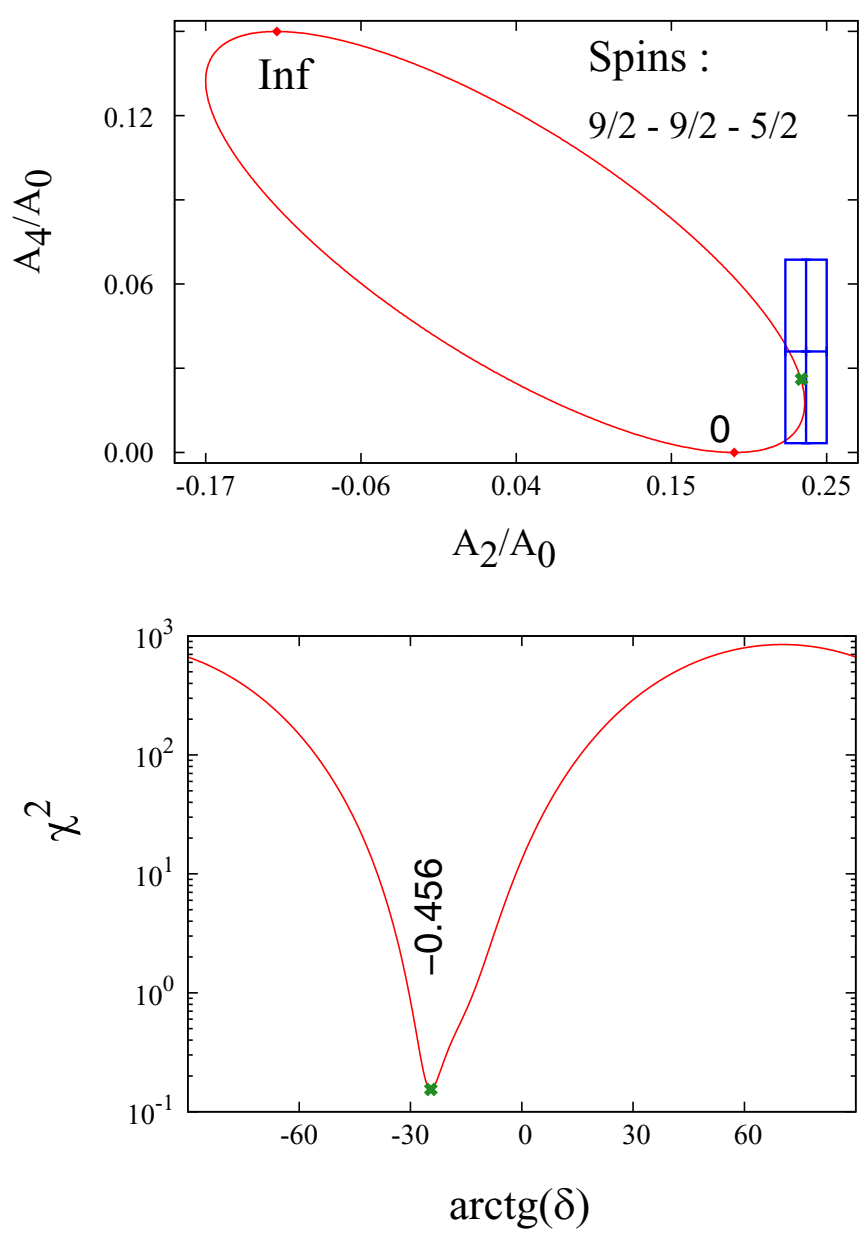

FIG. 4. Angular-correlation analysis for the $589-875 \mathrm{keV}$ cascade in ${ }^{87} \mathrm{Br}$. See text for more details.

A $\Delta I=2$ band based on the $\pi g_{9 / 2}$ configuration is expected to appear at relatively low energy in ${ }^{87} \mathrm{Br}$ with a bandhead spin-parity of $9 / 2^{+}$based on the deformed Nilsson scheme and also on the comparison with the level schemes of the neighboring odd-proton nuclei. In ${ }^{89} \mathrm{Rb}$ this bandhead is at $1195 \mathrm{keV}$ excitation energy [27]. In ${ }^{87} \mathrm{Br}$ the bandhead energy of Band 3 is close to this value. These arguments suggest $\pi g_{9 / 2}$ configuration to Band 3 and $9 / 2^{+}$spin-parity to its bandhead level. Indeed, $9 / 2^{+}$spin-parity has been firmly assigned to the $1464 \mathrm{keV}$ level in Ref. [4]. The observed stretched quadrupole character for the two lowest transitions of this band suggests $13 / 2^{+}$and $17 / 2^{+}$spin-parities for the second and third levels of the band, respectively. For the two highest levels of the band only tentative spin-parities of $\left(21 / 2^{+}\right)$and $\left(25 / 2^{+}\right)$could be assigned based on the $\Delta I=$ 2 band character. Reference [4] assigned $5 / 2^{-}$and $9 / 2^{-}$ spin-parities for the ground state and for the $975 \mathrm{keV}$ level, respectively. These assignments are in a good agreement with observed multipole characters of the 875 and $589 \mathrm{keV}$ transitions in the present experiment. The tentative spin-parity assignments of the other levels in Band 1 have been derived from the $\Delta I=1$ band character of this level structure. $3 / 2$ and $7 / 2$ spin values are assigned to the 6 and $801 \mathrm{keV}$ levels based on the observed multipolarities of the 663 and $796 \mathrm{keV}$ 


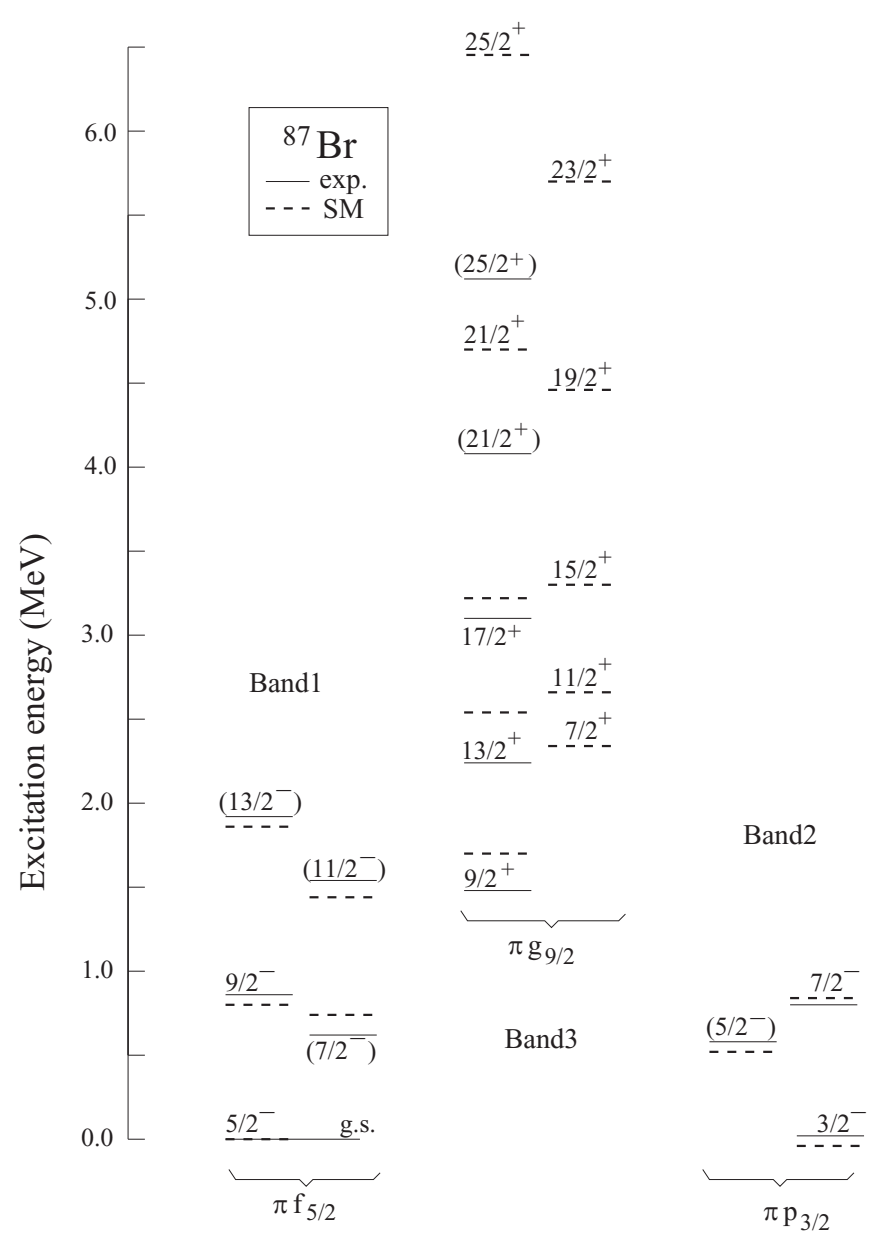

FIG. 5. Comparison of the observed (exp.) and calculated (SM) levels of ${ }^{87} \mathrm{Br}$.

transitions assuming that spin values increase with increasing level energy. Negative parity is assigned to these levels because no positive-parity states are expected at such low energy. The tentative $\left(5 / 2^{-}\right)$spin-parity of the $579 \mathrm{keV}$ level is assigned assuming $\Delta I=1$ band character for Band 2 . The tentative $\left(3 / 2^{-}\right)$spin-parity of the $333 \mathrm{keV}$ level is taken from Ref. [4].

\section{DISCUSSION}

The observed medium-spin band structures can qualitatively be understood based on the deformed, rotational picture. Indeed, in this picture two strongly coupled bands, based on the 5/2[303] $\left(\pi f_{5 / 2}\right)$ and $3 / 2$ [301] (mixed $\pi p_{3 / 2}$ and $\pi f_{5 / 2}$ ) Nilsson orbitals, and one decoupled band, based on the $1 / 2[440]\left(\pi g_{9 / 2}\right)$ Nilsson orbital, are expected to appear at low excitation energy with bandhead spin-parities of $5 / 2^{-}$, $3 / 2^{-}$, and $9 / 2^{+}$, respectively.

However, ${ }^{87} \mathrm{Br}$ with only two neutrons above the $N=50$ shell closure is expected to be a shell-model nucleus. Therefore, to verify the suggested spin-parities and configurations, we compared them with results of the contemporary shell model calculations using a large valence space including the $1 f_{5 / 2}, 2 p_{3 / 2}, 2 p_{1 / 2}, 1 g_{9 / 2}$ orbitals for protons and the $2 d_{5 / 2}$,
TABLE III. Proton and neutron occupations of excited states in ${ }^{87} \mathrm{Br}$ calculated using the shell model.

\begin{tabular}{|c|c|c|c|c|c|c|c|c|c|}
\hline \multirow[b]{2}{*}{$I^{\pi}$} & \multicolumn{5}{|c|}{ Neutrons } & \multicolumn{4}{|c|}{ Protons } \\
\hline & $d_{5 / 2}$ & $s_{1 / 2}$ & $g_{7 / 2}$ & $d_{3 / 2}$ & $h_{11 / 2}$ & $f_{5 / 2}$ & $p_{3 / 2}$ & $p_{1 / 2}$ & $g_{9 / 2}$ \\
\hline \multicolumn{10}{|c|}{ Band1 } \\
\hline $5 / 2^{-}$ & 1.61 & 0.13 & 0.07 & 0.12 & 0.08 & 4.48 & 1.97 & 0.33 & 0.23 \\
\hline $7 / 2^{-}$ & 1.59 & 0.21 & 0.04 & 0.11 & 0.05 & 4.52 & 1.96 & 0.33 & 0.19 \\
\hline $9 / 2^{-}$ & 1.73 & 0.11 & 0.03 & 0.09 & 0.04 & 4.57 & 1.90 & 0.31 & 0.22 \\
\hline $11 / 2^{-}$ & 1.79 & 0.06 & 0.01 & 0.11 & 0.03 & 4.63 & 1.87 & 0.28 & 0.21 \\
\hline $13 / 2^{-}$ & 1.85 & 0.05 & 0.07 & 0.06 & 0.02 & 4.64 & 1.87 & 0.28 & 0.20 \\
\hline \multicolumn{10}{|c|}{ Band2 } \\
\hline $3 / 2^{-}$ & 1.60 & 0.15 & 0.07 & 0.10 & 0.08 & 3.90 & 2.57 & 0.30 & 0.23 \\
\hline $5 / 2_{2}^{-}$ & 1.54 & 0.27 & 0.05 & 0.09 & 0.05 & 3.58 & 2.80 & 0.43 & 0.18 \\
\hline $7 / 2_{2}^{-}$ & 1.63 & 0.20 & 0.04 & 0.09 & 0.04 & 3.92 & 2.53 & 0.35 & 0.20 \\
\hline \multicolumn{10}{|c|}{ Band3 } \\
\hline $7 / 2^{+}$ & 1.56 & 0.13 & 0.06 & 0.11 & 0.14 & 3.97 & 1.68 & 0.30 & 1.05 \\
\hline $9 / 2^{+}$ & 1.57 & 0.14 & 0.08 & 0.14 & 0.07 & 4.03 & 1.57 & 0.28 & 1.12 \\
\hline $11 / 2^{+}$ & 1.73 & 0.08 & 0.03 & 0.10 & 0.06 & 4.08 & 1.53 & 0.30 & 1.09 \\
\hline $13 / 2^{+}$ & 1.56 & 0.22 & 0.04 & 0.13 & 0.04 & 3.90 & 1.68 & 0.31 & 1.11 \\
\hline $15 / 2^{+}$ & 1.75 & 0.09 & 0.02 & 0.04 & 0.10 & 4.37 & 1.36 & 0.22 & 1.05 \\
\hline $17 / 2^{+}$ & 1.78 & 0.05 & 0.02 & 0.09 & 0.07 & 4.30 & 1.41 & 0.22 & 1.08 \\
\hline $19 / 2^{+}$ & 1.22 & 0.03 & 0.03 & 0.05 & 0.67 & 4.66 & 1.59 & 0.24 & 0.51 \\
\hline $21 / 2^{+}$ & 1.76 & 0.06 & 0.03 & 0.12 & 0.03 & 3.82 & 1.83 & 0.29 & 1.07 \\
\hline $23 / 2^{+}$ & 1.74 & 0.02 & 0.02 & 0.04 & 0.18 & 4.48 & 1.46 & 0.14 & 0.91 \\
\hline $25 / 2^{+}$ & 1.31 & 0.02 & 0.58 & 0.05 & 0.03 & 3.84 & 1.83 & 0.26 & 1.07 \\
\hline
\end{tabular}

$3 s_{1 / 2}, 1 g_{7 / 2}, 2 d_{3 / 2}, 1 h_{11 / 2}$ orbitals for neutrons, outside the ${ }^{78} \mathrm{Ni}$ core.

The model and the parameters of the calculations are the same as used in Ref. [4]. The experimental and calculated level energies, spin-parities and single-particle configurations are plotted in Fig. 5.

It can be seen in the figure that the tentative experimental spin-parities are rather well reproduced by the calculations. The level energies are also relatively well reproduced for the states belonging to Band 1 and Band 2. There are, however, larger differences between the experimental and calculated level energies for the levels of Band 3, especially for its highest-energy levels. Adjusting the proton $g_{9 / 2}-$ neutron $d_{5 / 2}$ interaction in the model, the bandhead energy of Band 3 could be reproduced, however the energy spacing between the consecutive levels would not be changed, thus we cannot get good agreement between the experimental and calculated energies. It is worth mentioning that while in Bands 1 and 2 all the predicted levels have been observed up to the highest observed spins, in case of Band 3 only the $\alpha=+1 / 2$ signature branch is observed. This is probably due to the fact that the $\alpha=-1 / 2$ signature branch is strongly non-yrast, thus those levels have not been excited in the experiment. This assumption is in agreement with the calculations, which predict this signature branch to be shifted up by about $1 \mathrm{MeV}$. Table III shows the calculated proton and neutron occupations of the levels. They confirm the proposed single-particle configuration assignments.

The deviation between the measured and calculated level energies in Band 3 is probably due to a larger collectivity 


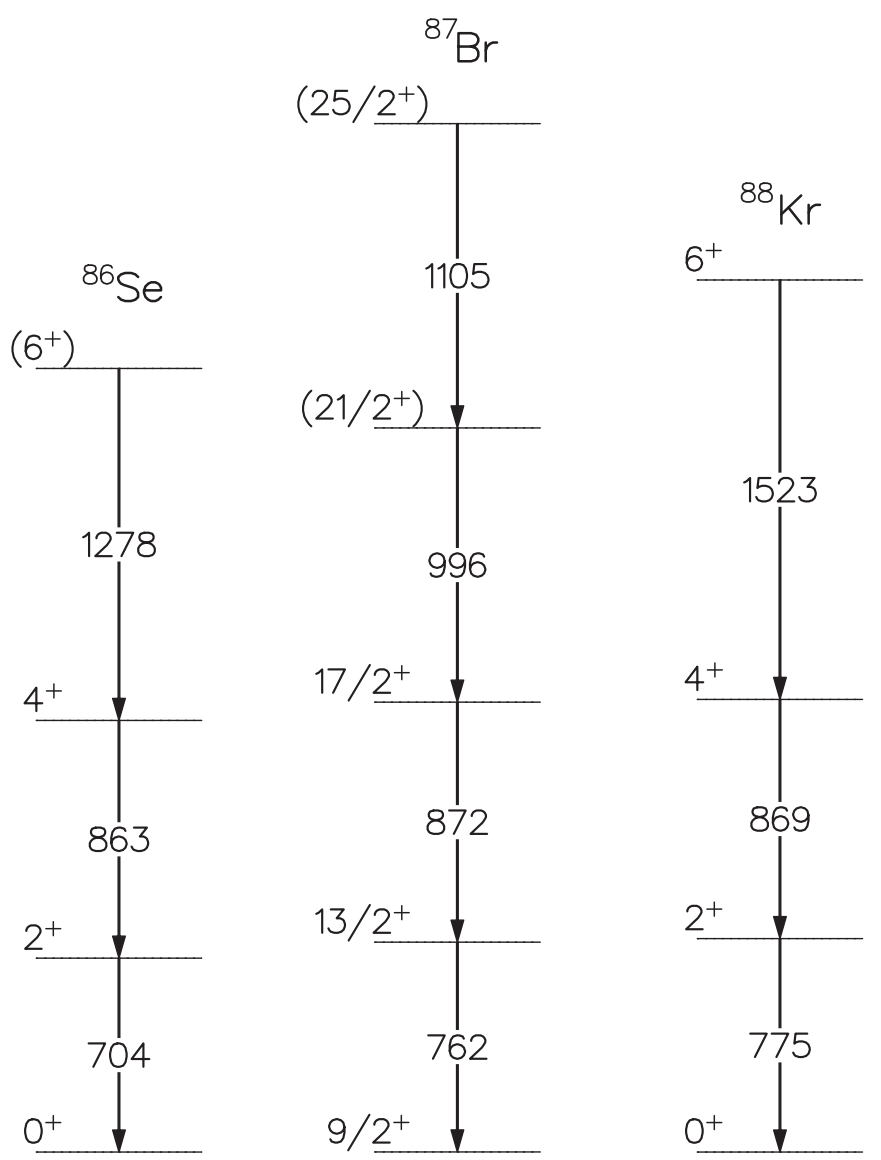

FIG. 6. Comparison of Band 3 in ${ }^{87} \mathrm{Br}$ with the ground-state bands of the neighboring $N=52$ even-even nuclei, ${ }^{86} \mathrm{Se}$ and ${ }^{88} \mathrm{Kr}$.

than that predicted by the calculations. The larger collectivity can be understood assuming that the proton in the $\pi g_{9 / 2}$ high- $j$ orbital polarizes the core. In this case, the quadrupolequadrupole interaction mixes other orbitals from the next shell, e.g., $2 d_{5 / 2}$, with the $g_{9 / 2}$ one in the configuration of Band 3. Such orbitals are not included in the model space of the present theory, thus the calculations cannot take the effect of them into account. This effect of the $g_{9 / 2}$ proton in increasing the collectivity seems to be supported also by the comparison of Band 3 with the ground-state bands of the also $N=52{ }^{86} \mathrm{Se}$ and ${ }^{88} \mathrm{Kr}$ nuclei. The data for the latter two bands were taken from Ref. [7]. This comparison is shown in Fig. 6, where the bandhead energy of the $\pi g_{9 / 2}$ band of ${ }^{87} \mathrm{Br}$ is shifted to match with that of the other two bands. It is seen that the levels of the $\pi g_{9 / 2}$ band are regularly spaced. Also, the energies of the higher-spin states are considerably lower than predicted by the shell model calculation, as seen in Fig. 5. These are characteristics for the increased collectivity. Contrarily, the levels of the ${ }^{86} \mathrm{Se}$ and ${ }^{88} \mathrm{Kr}$ bands, which have no $\pi g_{9 / 2}$ contribution in their configurations, are irregular and follow the shell model predictions as discussed in Ref. [7]. In fact, as discussed in Ref. [28] the $\pi g_{9 / 2}$ contribution in the configurations of these states is small but still exists.

\section{SUMMARY}

Medium-spin excited states of the neutron-rich ${ }^{87} \mathrm{Br}$ nucleus were studied by means of in-beam $\gamma$ spectroscopy of ${ }^{235} \mathrm{U}(n, f)$ fission fragments, using the EXILL Ge detector array. The fission was induced by the cold-neutron beam of the PF1B facility of the Institut Laue-Langevin, Grenoble. The medium-spin level scheme of this nucleus was built for the first time. The observed excited states form three bandlike structures. Angular correlation information from the experiment combined with systematics of the neighboring odd-proton nuclei enabled tentative spin-parity assignments to the new levels and configuration assignments to the bands. They can be understood as bands built on the $\pi f_{5 / 2}, \pi\left(p_{3 / 2}+\right.$ $f_{5 / 2}$ ), and $\pi g_{9 / 2}$ configurations. These assignments have been confirmed by the results of contemporary shell model calculations using a large valence space. ${ }^{87} \mathrm{Br}$ has $5 / 2^{-}$ground state spin-parity in contrast to the odd-mass $\mathrm{Br}$ isotopes containing fewer neutrons, which have $3 / 2^{-}$ground state spin-parity. Properties of the $\pi g_{9 / 2}$ band show an increased collectivity compared to the other bands in ${ }^{87} \mathrm{Br}$ and in the neighboring nuclei. This increased collectivity in this band can be understood assuming that the $\pi g_{9 / 2}$ high- $j$ proton polarizes the core.

\section{ACKNOWLEDGMENTS}

The authors thank the technical services staffs of the ILL, LPSC, and GANIL for supporting the EXILL campaign. The EXOGAM Collaboration and the INFN Legnaro are acknowledged for the loan of Ge detectors. This work was supported by the National Research, Development and Innovation Fund of Hungary, financed under the K18 funding scheme with Projects No. K128947 and No. K124810, as well as by the European Regional Development Fund (Contract No. GINOP-2.3.3-15-2016-00034). This work was also supported by the Polish National Science Centre under Contract No. DEC-2013/09/B/ST2/03485 and by the Bulgarian Ministry of Education and Science under the National Research Program "Young scientists and postdoctoral students." I.K. was supported by National Research, Development and Innovation Office-NKFIH, Contract No. PD 124717. D.L.B. and C.A.U. acknowledge support from the Extreme Light Infrastructure Nuclear Physics (ELI-NP) Phase II, a project cofinanced by the Romanian Government and the European Union through the European Regional Development Fund, the Competitiveness Operational Programme (1/07.07.2016, COP, ID 1334).
[1] R. Taniuchi et al., Nature (London) 569, 53 (2019).

[2] C. M. Shand et al., Phys. Lett. B 773, 492 (2017).

[3] B. M. Nyakó et al., J. Phys.: Conf. Ser. 724, 012051 (2016).
[4] J. Wiśniewski, W. Urban, M. Czerwinski, J. Kurpeta, A. Plochocki, M. Pomorski et al., Phys. Rev. C 100, 054331 (2019).

[5] M.-G. Porquet et al., Eur. Phys. J. A 28, 153 (2006). 
[6] A. Astier et al., Phys. Rev. C 88, 024321 (2013).

[7] M. Czerwinski et al., Phys. Rev. C 88, 044314 (2013).

[8] T. Materna et al., Phys. Rev. C 92, 034305 (2015).

[9] M. Czerwinski, T. Rzaca-Urban, W. Urban, P. Baczyk, K. Sieja, B. M. Nyakó et al., Phys. Rev. C 92, 014328 (2015).

[10] M. Czerwinski, T. Rzaca-Urban, W. Urban, P. Baczyk, K. Sieja, J. Timár et al., Phys. Rev. C 93, 034318 (2016).

[11] M. Czerwinski, K. Sieja, T. Rzaca-Urban, W. Urban, A. Plochocki, J. Kurpeta et al., Phys. Rev. C 95, 024321 (2017).

[12] T. Rzaca-Urban, M. Czerwinski, W. Urban, A. G. Smith, I. Ahmad, F. Nowacki, and K. Sieja, Phys. Rev. C 88, 034302 (2013).

[13] H. Abele et al., Nucl. Instrum. Methods A 562, 407 (2006).

[14] M. Jentschel et al., J. Instrum. 12, P11003 (2017).

[15] J. Simpson et al., Acta Phys. Hung. New Ser.: Heavy Ion Phys. 11, 159 (2000).

[16] D. Bazzacco et al., in Proceedings of the 5th International Seminar on Nuclear Physics, edited by A. Covello (World Scientific, Singapore, 1999), pp. 417-430.
[17] G. S. Simpson, J. C. Angelique, J. Genevey, J. A. Pinston, A. Covello, A. Gargano, U. Koster, R. Orlandi, A. Scherillo et al., Phys. Rev. C 76, 041303(R) (2007).

[18] A. A. Sonzogni, Nucl. Data Sheets 93, 599 (2001).

[19] J. Chen, Nucl. Data Sheets 146, 1 (2017).

[20] D. Abriola and A. A. Sonzogni, Nucl. Data Sheets 109, 2501 (2008).

[21] B. Singh, A. A. Rodionov, and Y. L. Khazov, Nucl. Data Sheets 109, 517 (2008).

[22] A. A. Sonzogni, Nucl. Data Sheets 103, 1 (2004).

[23] W. Urban et al., J. Instrum. 8, P03014 (2013).

[24] D. C. Radford, Nucl. Instrum. Methods A 361, 297 (1995).

[25] S. J. Zhu, J. H. Hamilton, A. V. Ramayya, M. G. Wang, J. K. Hwang, E. F. Jones et al., Phys. Rev. C 59, 1316 (1999).

[26] J. K. Hwang, A. V. Ramayya, J. Gilat, J. H. Hamilton, L. K. Peker, J. O. Rasmussen et al., Phys. Rev. C 58, 3252 (1998).

[27] B. Singh, Nucl. Data Sheets 114, 1 (2013).

[28] J. Litzinger et al., Phys. Rev. C 92, 064322 (2015). 